\title{
Experimental and computational analysis of the coolant distribution considering the viscosity of the cutting fluid during machining with helical deep hole drills
}

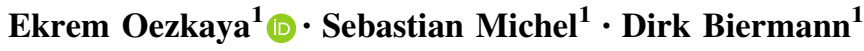

Received: 25 April 2021 / Revised: 13 June 2021 / Accepted: 18 November 2021/Published online: 15 February 2022

(C) The Author(s) 2022

\begin{abstract}
An experimental analysis regarding the distribution of the cutting fluid is very difficult due to the inaccessibility of the contact zone within the bore hole. Therefore, suitable simulation models are necessary to evaluate new tool designs and optimize drilling processes. In this paper the coolant distribution during helical deep hole drilling is analyzed with high-speed microscopy. Micro particles are added to the cutting fluid circuit by a developed high-pressure mixing vessel. After the evaluation of suitable particle size, particle concentration and coolant pressure, a computational fluid dynamics (CFD) simulation is validated with the experimental results. The comparison shows a very good model quality with a marginal difference for the flow velocity of $1.57 \%$ between simulation and experiment. The simulation considers the kinematic viscosity of the fluid. The results show that the fluid velocity in the chip flutes is low compared to the fluid velocity at the exit of the coolant channels of the tool and drops even further between the guide chamfers. The flow velocity and the flow pressure directly at the cutting edge decrease to such an extent that the fluid cannot generate a sufficient cooling or lubrication. With the CFD simulation a deeper understanding of the behavior and interactions of the cutting fluid is achieved. Based on these results further research activities to improve the coolant supply can be carried out with great potential to evaluate new tool geometries and optimize the machining process.
\end{abstract}

Ekrem Oezkaya

ekrem.oezkaya@tu-dortmund.de

1 Institute of Machining Technology, Dortmund University of Technology (TU Dortmund), Dortmund 44227, Germany
Keywords Computational fluid dynamics (CFD) analysis · Viscosity - Tracer micro particles $\cdot$ Fluid behavior $\cdot$ Helical deep hole drilling

\section{List of symbols}

$C_{\omega} \quad$ Cross-diffusions term

c Specific heat capacity, $\mathrm{J} /(\mathrm{kg} \cdot \mathrm{K})$

$D_{\omega} \quad$ Term of diffusion

$\mathrm{CD}_{\mathrm{k} \omega} \quad$ Positive part of the cross-diffusion

$d \quad$ Diameter, mm

$d_{\text {par }} \quad$ Particle diameter, $\mu \mathrm{m}$

$F_{1}, F_{2} \quad$ Blending function, of $k-\omega$-SST

$k \quad$ Turbulent kinetic energy, $\mathrm{m}^{2} / \mathrm{s}^{2}$

$k_{\text {par }} \quad$ Particle concentrations, g/L

$l \quad$ Length, $\mathrm{mm}$

l/d Length-to-diameter ratio

$l_{\mathrm{t}} \quad$ Drilling depth, $\mathrm{mm}$

$M \quad$ Molar mass, $\mathrm{g} / \mathrm{mol}$

$\dot{m} \quad$ Mass flow, $\mathrm{kg} / \mathrm{s}$

$n \quad$ Rotational speed, $\mathrm{r} / \mathrm{min}$

$P \quad$ Spindle power, $\mathrm{kW}$

$P_{\omega} \quad$ Production term

$\mathrm{p} \quad$ Pressure, $\mathrm{MPa}$

$r \quad$ Ration between fluid pressure and fluid density

$S_{i j} \quad$ Shear rate tensor, $1 / \mathrm{s}$

$s \quad$ Distance, $\mathrm{mm}$

$T \quad$ Temperature, ${ }^{\circ} \mathrm{C}$

TI Turbulence intensity

$t \quad$ Time, s

$u_{i}, u_{j} \quad$ Velocity vector, Cartesian

$u, v, w \quad$ Velocity component, $\mathrm{m} / \mathrm{s}$

$V \quad$ Volume, L

$V_{\text {RMS }}$ Turbulent velocity fluctuations

$v \quad$ Velocity, $\mathrm{m} / \mathrm{s}$

$v_{\mathrm{k}} \quad$ Kinematic viscosity, $\mathrm{m}^{2} / \mathrm{s}$ 
$v_{\mathrm{f}} \quad$ Feed rate, $\mathrm{mm} / \mathrm{min}$

$x_{i}, x_{j} \quad$ Component of the Cartesian position vector, $\mathrm{m}$

\section{Greek symbols}

$\alpha_{\omega}, \alpha_{1} \quad$ Model constants of $k-\omega$-SST

$\varepsilon \quad$ Dissipation rate of turbulent kinetic energy, $\mathrm{m}^{2} / \mathrm{s}$

$\epsilon_{\omega} \quad$ Destruction term

$\omega \quad$ Specific turbulent dissipation rate, $1 / \mathrm{s}$

$\rho \quad$ Density, $\mathrm{kg} / \mathrm{m}^{3}$

$\beta_{\omega}, \beta_{\mathrm{k}} \quad$ Model constants of $k-\omega$-SST

$\zeta \quad$ Flow value

$\mu \quad$ Dynamic viscosity, $\mathrm{kg} / \mathrm{ms}$

$\mu_{\mathrm{t}} \quad$ Turbulent viscosity, $\mathrm{kg} / \mathrm{ms}$

$\rho \quad$ Density, $\mathrm{kg} / \mathrm{m}^{3}$

$\sigma_{\mathrm{k}}, \sigma_{\omega} \quad$ Model constants of $k-\omega$-SST

$\tau_{i j}^{\text {mol }} \quad$ Momentum exchange, $\mathrm{kg} / \mathrm{ms}^{2}$

$\tau_{i j}^{\text {RANS }}$ Reynold-stress tensor, $\mathrm{m}^{2} / \mathrm{s}^{2}$

$\lambda$ Thermal conductivity, $\mathrm{W} /\left(\mathrm{m} \cdot \mathrm{K}^{-1}\right)$

\section{Subscript}

$A, B$ Cutting plane

exp Experiment

$i, j \quad$ Direction of the component

max Maximum

sim Simulation

tanh Hyperbolic tangent

\section{Mathematical operations}

a Partial differential

- Temporal averaging

$\sim$ Fluctuation value

$\triangle \quad$ Delta

$\emptyset \quad$ Model coefficients

\section{Introduction}

Drilling is one of the most common and fundamental machining processes, with about $75 \%$ of the bore holes being produced with helical drills. Numerous studies have been carried out to optimize cutting parameters and tool geometries in order to improve the cutting performance. Drilling with helical drills has many applications, e.g., in the aerospace industry, where millions of holes are required for the assembly of aircraft structures [1]. The design of an efficient and powerful drill incorporates numerous conflicts such as low cutting forces, wear resistance, torsional and axial stability and chip evacuation capability [2]. In addition to experimental studies, researchers have used analytical mathematic and numerical approaches to analyze the drilling process. Sophisticated hybrid modeling techniques and artificial intelligence (AI) are also used to describe the complex process and calculate feed forces, torques, temperatures and tool wear [3-7]. However, most of these studies focus on the calculation of fundamental physical variables and use 2D simulations with their associated simplifications [8]. Due to continually increasing industrial requirements for efficient machining, it is necessary to develop sophisticated 3D modeling methods. Thus, the drilling process can be analyzed adequately to understand the complex correlations of workpiece, tool and cutting fluid [7].

Compared to milling or turning, the cutting zone for drilling tools is enclosed and located inside the workpiece, which leads to increased thermo-mechanical loads [9]. The mechanical energy supplied during the machining process is completely converted into heat and distributed in the chip, the tool and the workpiece [10]. The cutting speed in drilling operations is low, but the continuous engagement of the cutting edge in combination with the removal of the chips through the drill flutes requires an efficient supply of cooling lubricant [11]. However, since the cutting zone is located inside the workpiece, it is challenging to provide a sufficient cooling lubricant supply directly to the highly loaded contact zone at the cutting edge [12]. Especially for the drilling of difficult-to-cut materials such as Inconel 718 optimal cooling conditions are absolutely necessary to counteract the material's high heat resistance, the low thermal conductivity and the tendency to work hardening [13]. Otherwise, these properties lead to insufficient tool performance, poor bore hole quality and high tool wear, and thus high manufacturing costs [14]. In order to achieve the desired productivity of the process and the required product quality, continuous improvements and new technological developments are required.

A drilling process is considered as a deep hole drilling process if the length-to-diameter ratio of the bore hole exceeds $l / d=10$ [15]. The most important processes are boring and trepanning association (BTA) deep hole drilling, single-lip deep hole drilling and helical deep hole drilling [16]. These processes are used, for example, for the machining of engine components in the automotive industry, for turbine and rocket engine parts in the aerospace industry or for parts in the medical industry. In helical deep hole drilling standard tools can produce bore holes with approx. $l / d=30-40$, special tools can reach even higher drilling depth of up to $l / d=40-70$ [17]. Compared to the standard tools, the load on special tools increases up to three to five times [9]. The process becomes even more difficult and complex with increasing depth due to vibrations, drill deflections and the heat build-up at the cutting edge [18]. Volz et al. [19] demonstrated that the width of the guide chamfers in particular could have an influence on the quality of the bore holes in helical deep hole drilling. The lack of guidance of the tool with narrow 
guide chamfers and the generation of lateral vibration led to a significant deterioration in roundness. Kirschner et al. [20] demonstrated that the flow of cooling lubricant during single-lip deep hole drilling also influenced chip breaking in addition to the transport function of the fluid for the chips. When machining Inconel 718 with single-lip deep hole drills, the chip breakage was forced by the larger cross-sectional area of spiral chips in the chip flute due to higher forces on the chips caused by the flowing coolant [20]. In the development of modern deep hole drilling machines, the design and construction of the cooling lubricant supply system to the supply deep hole drilling oil to the drilling process therefore plays an important role. In addition to special pumps with high pressures and controllable volume flows, the filtering and cooling of the deep hole drilling oil also has an influence on the efficiency of the coolant supply [21]. The supply of cooling lubricant is of particular relevance for deep hole drilling processes, in addition to the cooling of the cutting edges, since the removal of chips is critical for process reliability [22]. In addition, a jamming of the chips in the chip flutes can lead to increased torque, which on the one hand accelerates tool wear and on the other hand can cause premature tool failure [23]. Suitable cutting parameters to produce process-favorable short-breaking chips [24] and the precise control of the cooling lubricant $[25,26]$ therefore play an important role to prevent insufficient bore hole quality and excessive tool wear [23, 27, 28]. In studies by Wegert et al. [29], a lack of cooling lubricant supply led to chipping of the cutting edges of single-lip deep hole drills. However, Wegert et al. [29] observed an influence of the cooling lubricant supply on the temperature development in the subsurface zone of the bore hole wall. The temperature of the subsurface zone, which is influenced by the cooling lubricant supply, is also the focus of Schmidt's current investigations. By adapting process parameters, it was possible to deliberately influence the condition of the peripheral layer of the bore wall in BTA deep hole drilling through the temperature in the area of the guide pads and the introduction of residual compressive stresses [30].

Computational fluid dynamics (CFD) simulations offer new possibilities to analyze and optimize the cooling lubricant supply. Compared to the established finite element method (FEM) simulations, CFD simulations are a relatively new field of research for machining processes. Especially for processes depending on the cooling lubricant supply it is necessary to developed future-oriented simulation models, to understand the fluid behavior and the interaction of fluid and machining process. Validated against experimental data, these simulation models enable rapid analysis of new tool geometries and help to optimize the drilling processes. Therefore, in this paper the flow behavior of the cooling lubricant during helical deep hole drilling is analyzed by experimental investigations as a basis to develop and validate the simulation model (see Fig. 1).

In comparison to conventional analysis, in which the properties of water are usually used to describe the cooling lubricant, the simulation in this paper takes into account the viscosity of the deep drilling oil. The simulation also uses the reynolds-averaged-navier-stokes (RANS) $k$ - $\omega$-SST turbulence model. Previous work has shown that this turbulence model is best suited for the CFD simulation of helical deep hole drilling processes with respect to computing time and simulation accuracy [31]. Conventional experimental setups to measure the flow behavior can hardly be used for the analysis of the cooling lubricant flow. The bore hole wall and the rotation of the drill make the contact zone inaccessible for the measurement equipment during helical deep hole drilling. The particle image velocimetry (PIV) technique uses pulsed laser light and micro-particles in the fluid to calculate flow velocities. It usually requires a stationary test setup without rotating parts such as the drill and a constant accessibility of the flow for the illumination with the laser light. The flow field is calculated by the analysis of the particle movement between two pictures taken in a short period of time $[32,33]$. Experimental investigations of the cutting fluid flow to validate the simulation are carried out with a special test setup using high-speed microscopy and a workpiece sample placed in transparent acrylic glass. Microparticles are inserted into the cutting fluid circuit via a high-pressure mixing vessel. Similar to the technique used in sophisticated PIV test setups, it is possible to track single micro-particles in a frame-by-frame analysis to calculate local flow velocities with this simple technique.

\section{Turbulence model}

CFD methods solve equations of motion and interaction between solid and fluid bodies using the Euler equations for non-viscous fluids and the Navier-Stokes equations for viscous fluids [34-36]. The deviations of the transport equations required for the RANS $k-\omega$-SST turbulence model can be described by the Navier-Stokes equations of the momentary values of a Newtonian incompressible fluid with

$$
\begin{aligned}
& \frac{\partial u_{i}}{\partial x_{i}}=0, \\
& \frac{\partial u_{j}}{\partial t}+\frac{\partial u_{i} u_{j}}{\partial x_{i}}=-\frac{\partial r}{\partial x_{j}}-\frac{\partial \tau_{i j}^{\mathrm{mol}}}{\partial x_{i}},
\end{aligned}
$$




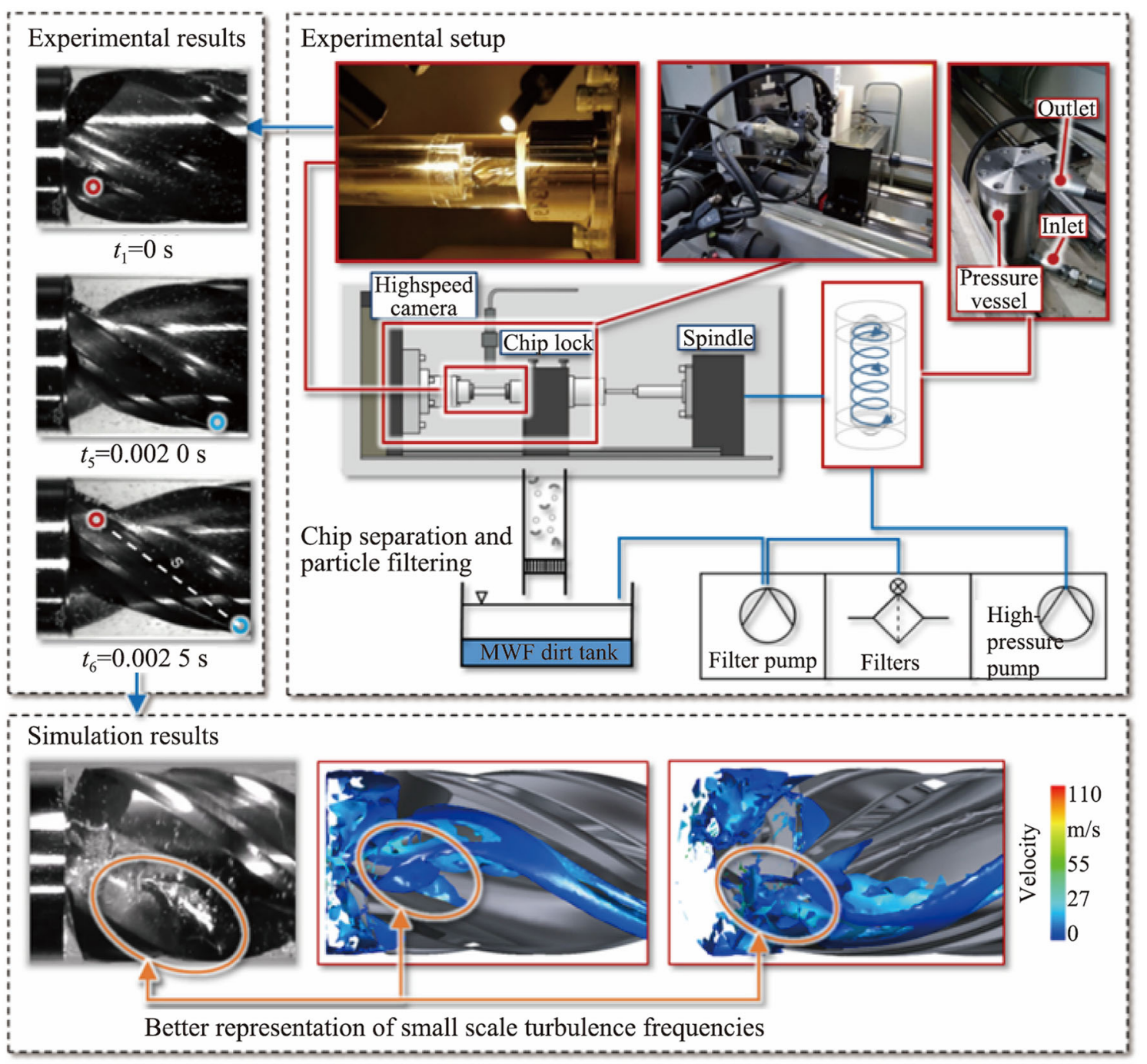

Fig. 1 Methodology

$\tau_{i j}^{\mathrm{mol}}=-2 v_{\mathrm{k}} S_{i j}$ mit $S_{i j}=\frac{1}{2}\left(\frac{\partial u_{i}}{\partial x_{j}}+\frac{\partial u_{j}}{\partial x_{i}}\right)$,

where Eq. (1) is the continuity equation, Eq. (2) the momentum equation and Eq. (3) the energy equation. The velocity component is $u, x$ the Cartesian coordinate, $r$ the ratio between the fluid pressure and fluid density, $t$ the time, $v_{\mathrm{k}}$ the kinematic viscosity, $S_{i j}$ the strain rate tensor and $\tau_{i j}^{\mathrm{mol}}$ the momentum exchange caused by the molecules. The momentary values of a statistical steady turbulent flow are divided into a time-mean value $\bar{\zeta}$ and a fluctuating part $\zeta^{\prime}$ and are defined for any flow value of $\zeta$ with

$\zeta=\bar{\zeta}+\zeta^{\prime}$.

The averaged flow value can be derived from the combination of the Navier-Stokes and Reynolds postulates $\frac{\partial \bar{u}_{i}}{\partial x_{i}}=0$,

$\frac{\partial \bar{u}_{j}}{\partial t}+\frac{\partial \bar{u}_{i} \bar{u}_{j}}{\partial x_{i}}=-\frac{\partial \bar{r}}{\partial x_{j}}-\frac{\partial \bar{\tau}_{i j}^{\mathrm{mol}}}{\partial x_{i}}-\frac{\partial \tau_{i j}^{\mathrm{RANS}}}{\partial x_{i}}$.

The separation of the flow variables leads to a special non-linear, convective term in the momentum conservation equation for which the averaging procedure creates another term [37]

$\overline{u_{i} u_{j}}=\overline{\left(u_{i}+u_{i}^{\prime}\right)\left(\bar{u}_{j}+u_{j}^{\prime}\right)}=\bar{u}_{i} \bar{u}_{j}+\underbrace{\overline{u_{i}^{\prime} u_{j}^{\prime}}}_{\tau_{i j}^{\text {RANS }}}$.

The Reynold-stress tensor $\tau_{i j}^{\text {RANS }}$ describes the momentum exchange and is based on the velocity fluctuations in the flow field. Although this tensor is symmetrical, the Eqs. (5) and (6) do not result in a closed system for the four dependent variables $\left(\bar{u}_{i}, \bar{r}\right)$ and the six additional 
independent unknowns of the Reynold-stress tensor $\left(\tau_{i j}^{\text {RANS }}\right)$ [38]. The problem cannot be solved by setting up new transport equations for the Reynold-stress tensor, since unknown terms with derivatives of the fluctuations of higher order occur repeatedly. The main task of turbulence modeling is the approximation of these terms to attribute the unknown terms to known correlations in order to close the equation system. CFD software utilizes transport equations which provide field of turbulence variables that can be grouped into scalar dependent variables and tensor variables [39].

The $k-\omega$ based shear stress transport (SST) model developed by Menter [40] is one of the most reliable RANS turbulence models and is implemented in the commercial ANSYS CFX solver. This model combines the advantages of $k-\varepsilon$ and $k-\omega$ models. The SST model employs the Wilcox $k$ - $\omega$ model [41] near the surface and the $k-\varepsilon$ model in the free-shear layers. The two models are bridged with an adopted blending function. The transport equation of the turbulent kinetic energy $k$ in the SST model is defined as

$$
\begin{aligned}
\frac{\partial(\bar{\rho} \hat{k})}{\partial t}+\frac{\partial}{\partial x_{j}}\left(\bar{\rho} \tilde{u_{j}} \tilde{k}\right)= & -\bar{\rho} \sim \sim_{i j}^{\text {RANS }} \frac{\partial \tilde{u}_{i}}{\partial \tilde{x}_{i}}-\beta_{\mathrm{k}} \bar{\rho} \tilde{k} \omega \\
& +\frac{\partial}{\partial x_{j}}\left(\left(\bar{\mu}+\sigma_{\mathrm{k}} \mu_{\mathrm{t}}\right) \frac{\partial \tilde{k}}{\partial x_{j}}\right),
\end{aligned}
$$

where $\rho$ is the density, $t$ the time, $x_{i}$ and $x_{j}$ the components of the cartesian position vector, $\mu$ the dynamic turbulent viscosity and $\mu_{\mathrm{t}}$ the turbulent viscosity. The specific dissipation rate $\omega$ is calculated with Eq. (9) and is composed of the $\omega$-production term of turbulent kinetic energy $P_{\omega}$, the destruction term $\epsilon_{\omega}$, part of the diffusion term $D_{\omega}$ and the cross-diffusion term $C_{\omega}$, which occurs during the transformation of the $\in$ equation into the $\omega$ notation.

$$
\begin{gathered}
\frac{\partial(\bar{\rho} \omega)}{\partial t}+\frac{\partial}{\partial x_{j}}\left(\bar{\rho} \tilde{u}_{j} \omega\right)=\underbrace{-\frac{\alpha_{\omega}}{\mu_{\mathrm{t}}}-\frac{\alpha_{\omega}}{\mu_{\mathrm{t}}} \bar{\rho} \tilde{R}_{i j} \frac{\partial \tilde{u}_{i}}{\partial \tilde{x}_{j}}}_{P_{\omega}}-\underbrace{\beta_{\omega} \bar{\rho} \omega^{2}}_{\epsilon_{\omega}} \\
+\frac{\partial}{\partial x_{j}}(\underbrace{\left(\bar{\mu}+\sigma_{\omega} \mu_{\mathrm{t}}\right) \frac{\partial \omega}{\partial x_{j}}}_{D_{\omega}})+\underbrace{2\left(1-F_{1}\right) \sigma_{\omega}^{(\epsilon)} \frac{\bar{\rho}}{\omega} \frac{\partial \tilde{k} \partial \omega}{\partial x_{j} \partial x_{j}}}_{C_{\omega}} .
\end{gathered}
$$

The empirical model coefficient $\emptyset=\left\{\alpha_{\omega}, \beta_{\omega}, \sigma_{\mathrm{k}}, \sigma_{\omega}\right\}$ is determined by blending a lower limit $\emptyset^{(\omega)}$ and an upper limit $\emptyset^{(\epsilon)}$ with

$\emptyset=F_{1} \emptyset^{(\omega)}+\left(1-F_{1}\right) \emptyset^{(\epsilon)}$.

With Eq. (10) the SST model calculates the value for the transition between areas near the surface and in the freeshear layers. The values of the model coefficients are listed in Table 1.
The blending function $F_{1}$ is determined by taking the wall distance $y$ and the dynamic viscosity $\mu$ into consideration and is defined as

$F_{1}=\tanh \left(\min \left(\max \left(\frac{\sqrt{\tilde{k}}}{\beta_{\mathrm{k}} \omega y}, \frac{500 \bar{\mu}}{\bar{\rho} \omega y^{2}}\right),\left(\frac{4 \bar{\rho} \sigma_{\omega}^{(\epsilon)} \hat{k}}{\mathrm{CD}_{\mathrm{k} \omega} y^{2}}\right)\right)^{4}\right)$.

The positive part of the cross-diffusion term is defined as $\mathrm{CD}_{\mathrm{k} \omega}$. It results from the transfer of the $k-\varepsilon$ equation into the $k-\omega$ model and is calculated with

$\mathrm{CD}_{\mathrm{k} \omega}=\max \left(\frac{2 \rho_{\omega}^{(\epsilon)}}{\omega} \frac{\partial \tilde{k}}{\partial x_{j}} \frac{\partial \omega}{\partial x_{j}}, 10^{-10}\right)$.

Near the surface the blending function moves towards the value of $1\left(F_{1}=1\right)$, increasing the importance of the $k$ $\omega$ formula. In the free-shear layers the blending function tends towards zero $\left(F_{1}=0\right)$ and favors the $k$ - $\varepsilon$ formula. By calculating the turbulent viscosity $\mu_{\mathrm{t}}$ with Eq. (13) the RANS $k-\omega$-SST model avoids the calculation of highly turbulent kinetic energy in the area of stagnation points in the flow.

$\mu_{\mathrm{t}}=\frac{\bar{\rho} \alpha_{1} \tilde{k}}{\max \left(\alpha_{1} \omega, \Omega F_{2}\right)}$.

The second blending function $F_{2}$ uses the remaining model coefficients $\beta_{\mathrm{k}}=0.09$ and $\alpha_{1}=0.31$ and is defined with

$F_{2}=\tanh \left(\max \left(\frac{2 \sqrt{\tilde{k}}}{\beta_{\mathrm{k}} \omega y}, \frac{500 \bar{\mu}}{\bar{\rho} \omega y^{2}}\right)\right)$.

\section{Experimental setup including a high-pressure mixing vessel}

The cutting fluid flow is visualized with micro-particles. To avoid a contamination of the whole cutting fluid circuit of the machine with these particles, a high-pressure mixing vessel was designed. Thus, the particles can be inserted into the pressurized oil stream just before the spindle. A filtering fleece with an appropriate filtration grade filters the oil and particle mixture collected in the chip lock. The

Table 1 SST Model constants

\begin{tabular}{lllll}
\hline & $\alpha_{\omega}$ & $\beta_{\omega}$ & $\sigma_{\mathrm{k}}$ & $\sigma_{\omega}$ \\
\hline Lower limit $\emptyset^{(\omega)}$ & 0.5556 & 0.0750 & 0.85 & 0.500 \\
Upper limit $\emptyset^{(\epsilon)}$ & 0.4400 & 0.0828 & 1.00 & 0.856 \\
\hline
\end{tabular}


inner volume of the mixing vessel amounts to $V=0.63 \mathrm{~L}$ and a maximum internal pressure of $p=10 \mathrm{MPa}$ was assumed for the design. The inlet and outlet were positioned on opposing sides to ensure a rotational fluid movement within the vessel, as well as an even distribution of the micro-particles in the cutting fluid (see Fig. 2).

The manufactured mixing vessel is integrated into the coolant circuit with high-pressure hoses between the highpressure pump and the spindle. The tracer micro-particles made by LaVision can thus be fed into the cutting fluid while avoiding a possible contamination of other machine components. The experimental investigations were carried out on a deep hole drilling machine TBT ML-200 (see Fig. 3). The workpiece material AISI 316L was machined with a solid carbide helical deep hole drill with a diameter of $d=5 \mathrm{~mm}$ and a TiAlN coating. The cutting fluid Swisscut Ortho NF-X 10 from Motorex has a kinematic viscosity of $v_{\mathrm{k}}=10.9 \mathrm{~mm}^{2} / \mathrm{s}\left(40^{\circ} \mathrm{C}\right)$. To observe the behavior of the cutting fluid during the drilling process, the workpieces were placed in transparent acrylic glass carriers replacing the borehole wall. The high-speed videos were recorded with a KEYENCE VW9000 high-speed camera, which offers a maximum frame rate of 230000 frams/s. To increase the image quality, additional lighting was used in the recording area. The lens of the high-speed camera was focused on the cutting zone and the process was recorded with a frame rate of 10000 frames/s and a resolution of 320 $\times 240$ pixels.

\section{Determination of suitable particle parameters and experimental results}

The polyamide micro-particle diameter $\left(d_{\mathrm{par}}=20 \mu \mathrm{m}, 55\right.$ $\mu \mathrm{m}, 100 \mu \mathrm{m})$ as well as the particle concentration $\left(k_{\text {par }}=0.5 \mathrm{~g} / \mathrm{L}, 1 \mathrm{~g} / \mathrm{L}, 2 \mathrm{~g} / \mathrm{L}\right.$ ) were varied in three stages to determine their respective traceability. The particle diameter $d_{\text {par }}=20 \mu \mathrm{m}$ was too small for a clear tracking in the high-speed recordings with a resolution of $320 \times 240$ pixels. The particle diameter $d_{\text {par }}=100 \mu \mathrm{m}$ showed great potential for detection and tracking of individual particles. However, large particle agglomerations were evident in the coolant flow. The best results were achieved with a particle diameter of $d_{\mathrm{par}}=55 \mu \mathrm{m}$ and a particle concentration of $k_{\text {par }}=2 \mathrm{~g} / \mathrm{L}$. The cutting fluid pressure was set to $p=8$ $\mathrm{MPa}$ since this corresponds to the usual industrial application (see Fig. 4).

The flow of the cutting fluid was first investigated with a non-rotating tool, whereby the high-speed recordings were examined frame by frame to detect and trace individual micro-particles. The tracked particles for a distance between the drill tip and the bore hole ground of $s=1 \mathrm{~mm}$ are marked in different colors in Fig. 5. The resulting flow lines and vortexes are presented for this tool position and for the position with full engagement of the drill. The occurring turbulences between the tool and the workpiece are increasing when the rotation of the helical drill is taken into account additionally.

The flow velocity in the flutes is too high to be detected with the limited frame rate of the high-speed camera at a suitable resolution. The lowest flow velocity is expected to be between the guide chamfers of the tool. Therefore, the average flow velocity was measured in this area. In order to calculate the velocity, individual micro-particles were selected and tracked frame by frame over a period of $\Delta t=$ $0.0025 \mathrm{~s}$ to determine the travelled distance (see Fig. 6). As soon as the tool engages the workpiece material and starts cutting, an increased momentum of the fluid can be detected. The average flow velocity over a distance of approximately $s \approx 6.26 \mathrm{~mm}$ was $v_{\text {oil }}=2.504 \mathrm{~m} / \mathrm{s}$.

\subsection{Boundary conditions and meshing}

The fluid model represents the internal geometry between the workpiece and the tool and requires an exact volume modeling. The flow area is relatively small and has an extremely high geometric complexity. The engagement position of the solid bodies and the fluid bodies must be differentiated in order to subsequently define the contact interfaces. The meshing process plays an important role in CFD simulations as it strongly influences the quality of the flow area mapping and thus the accuracy of the results. This aspect is often underestimated due to the fact that CFD simulations are not yet as well established in machining technology as FEM simulations. The meshing strategy in CFD is associated with considerably more effort and difficulties, especially for the small dimensions and the complex tool geometries of helical deep hole drilling.

The entire internal flow area must be accurately mapped to ensure the continuity of the fluid body to guarantee accurate calculations of flow pressure and the flow velocity in the fluid. The flow velocity depends on the Reynolds number. Very high velocity gradients occur near the wall, requiring an even finer mesh of the already very small and complex areas. These so-called boundary layers (inflation layers) often require manual meshing, i.e., the element size of the grids and the arrangement of the grids must be controlled manually. Therefore, the number of elements for the whole fluid body can amount to several millions. The numerical calculations to solve the coupled differential equation systems of the conservation equations (mass, momentum, energy conservation) require high-performance computer systems. Compared to 2D meshing, 3D meshing requires, e.g., an elliptical grid generation so that the partial differential equations can be transferred to the respective mesh coordinates. Many different parameters 

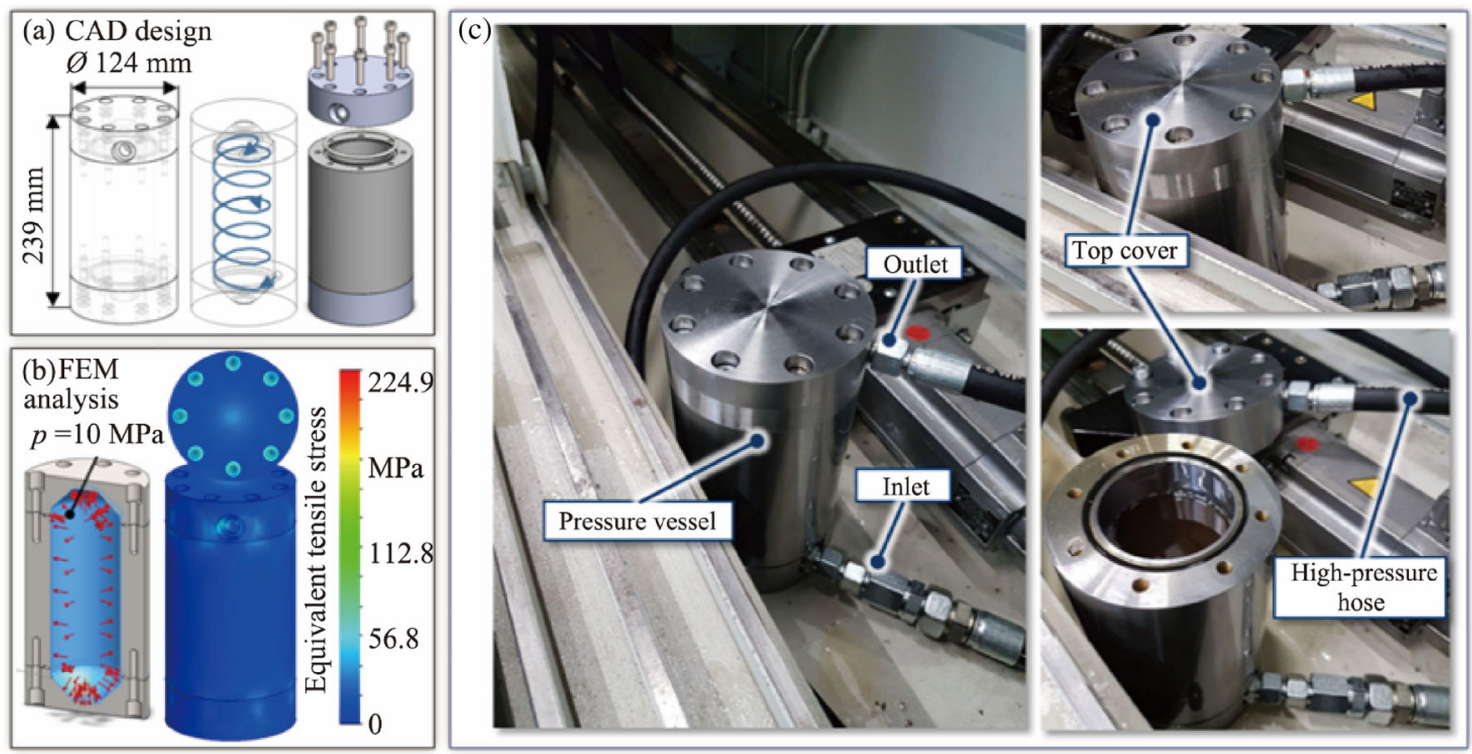

Fig. 2 a CAD design of the high-pressure mixing vessel, b FEM analysis of the high-pressure mixing vessel, $\mathbf{c}$ integration of the high- pressure mixing vessel

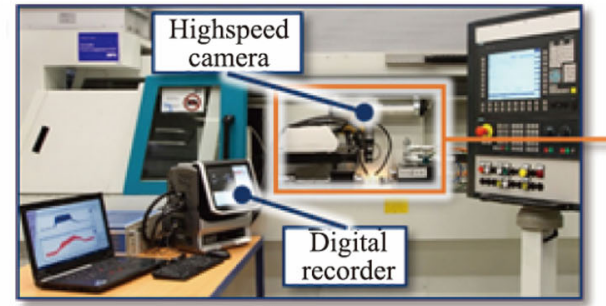

(a)

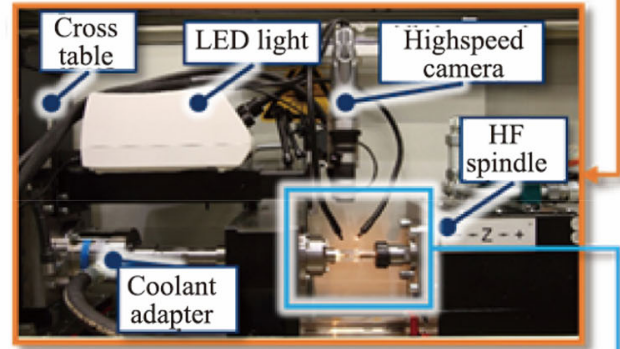

(b)

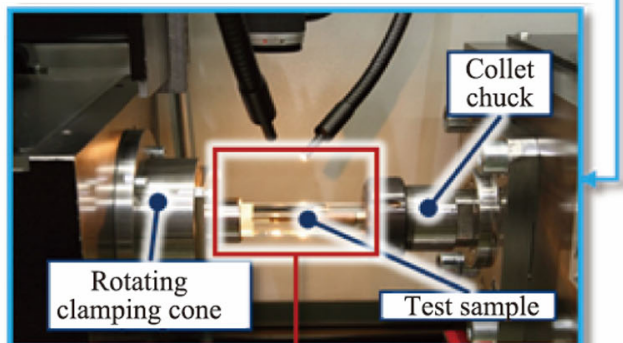

(c)
Deep hole drilling machine TBTML-200

Diameter range: $\quad d=0.5-6 \mathrm{~mm}$

Spindle power: $\quad P=4 \mathrm{~kW}$

Rotational speed: $\quad n_{\max }=36.0001 / \mathrm{min}$

Feed rate: $\quad V_{\text {fmac }}=15.000 \mathrm{~mm} / \mathrm{min}$

Drilling depth: $\quad I_{\text {tmax }}=450 \mathrm{~mm}$

Cooling lubricant: $\quad$ Motorex Ortho NF-X

Pressure: $\quad p_{\max }=23 \mathrm{MPa}$

Force measurement: Kistler Typ 9272

Charge amplifier: Kistler Typ 5011

Keyence VW-9000 high speed camera

Resolution: $\quad 640 \times 480$ pixels

Frame rate: $\quad \max .230000$ frame/s

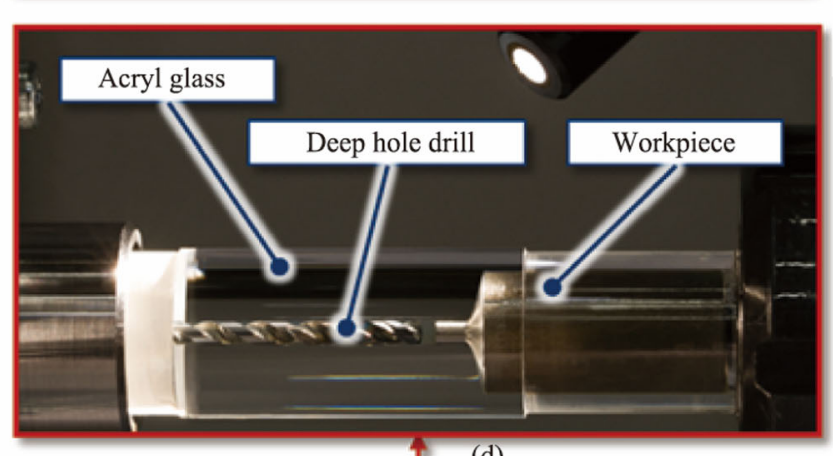

(d)

Fig. 3 Experimental setup a deep hole drilling machine TBT ML-200 with high-speed camera setup, b detailed view of the experimental setup, c clamping of the test sample, $\mathbf{d}$ workpiece and deep hole drill placed into transparent acrylic glass 


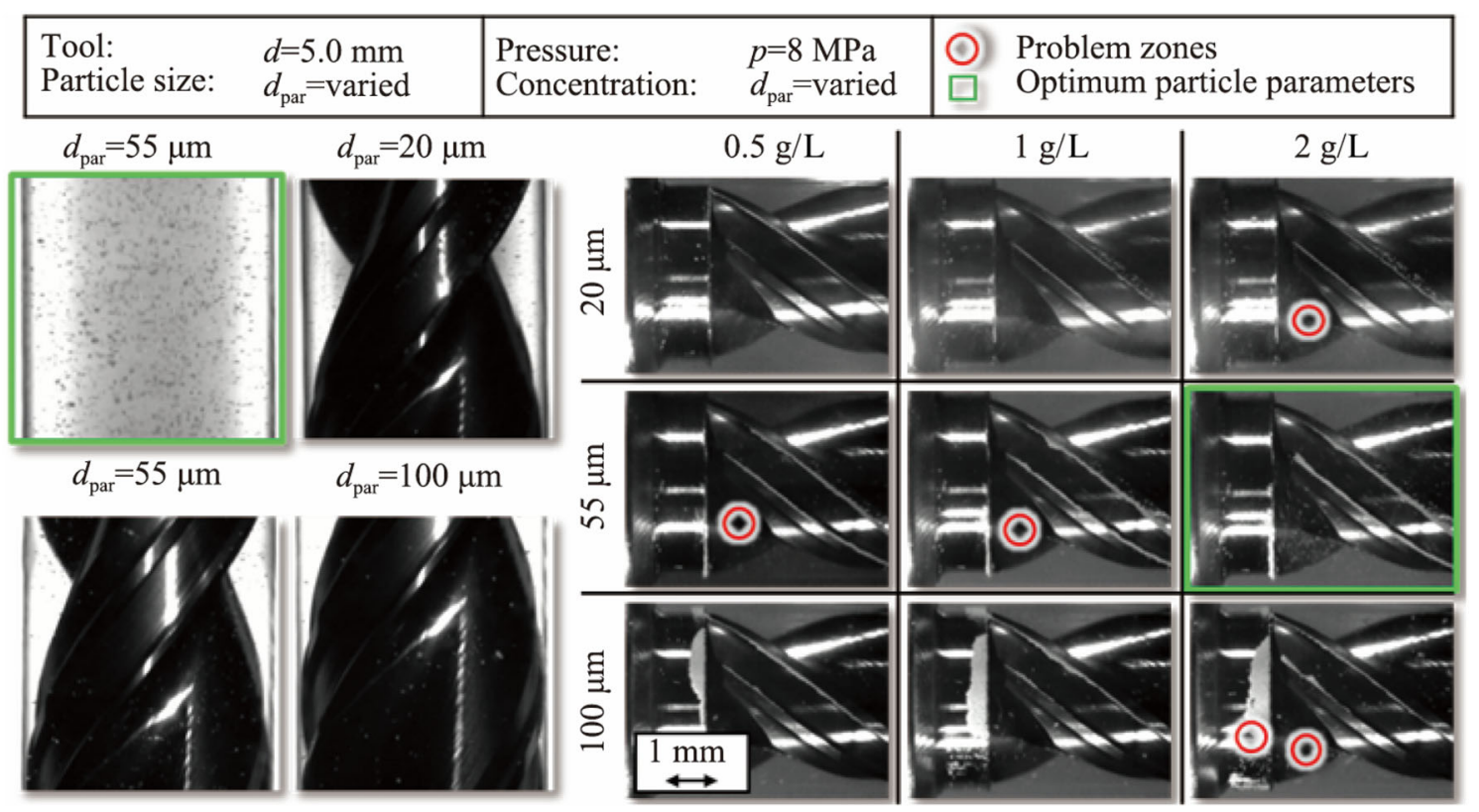

Fig. 4 Comparison of polyamide micro-particle sizes $d_{\text {par }}(20 \mu \mathrm{m}, 55 \mu \mathrm{m}, 100 \mu \mathrm{m})$ and particle concentration $k_{\text {par }}(0.5 \mathrm{~g} / \mathrm{L}, 1 \mathrm{~g} / \mathrm{L}, 2 \mathrm{~g} / \mathrm{L})$; best results were achieved with $d_{\text {par }}=55 \mu \mathrm{m}$ and $k_{\text {par }}=2 \mathrm{~g} / \mathrm{L}$

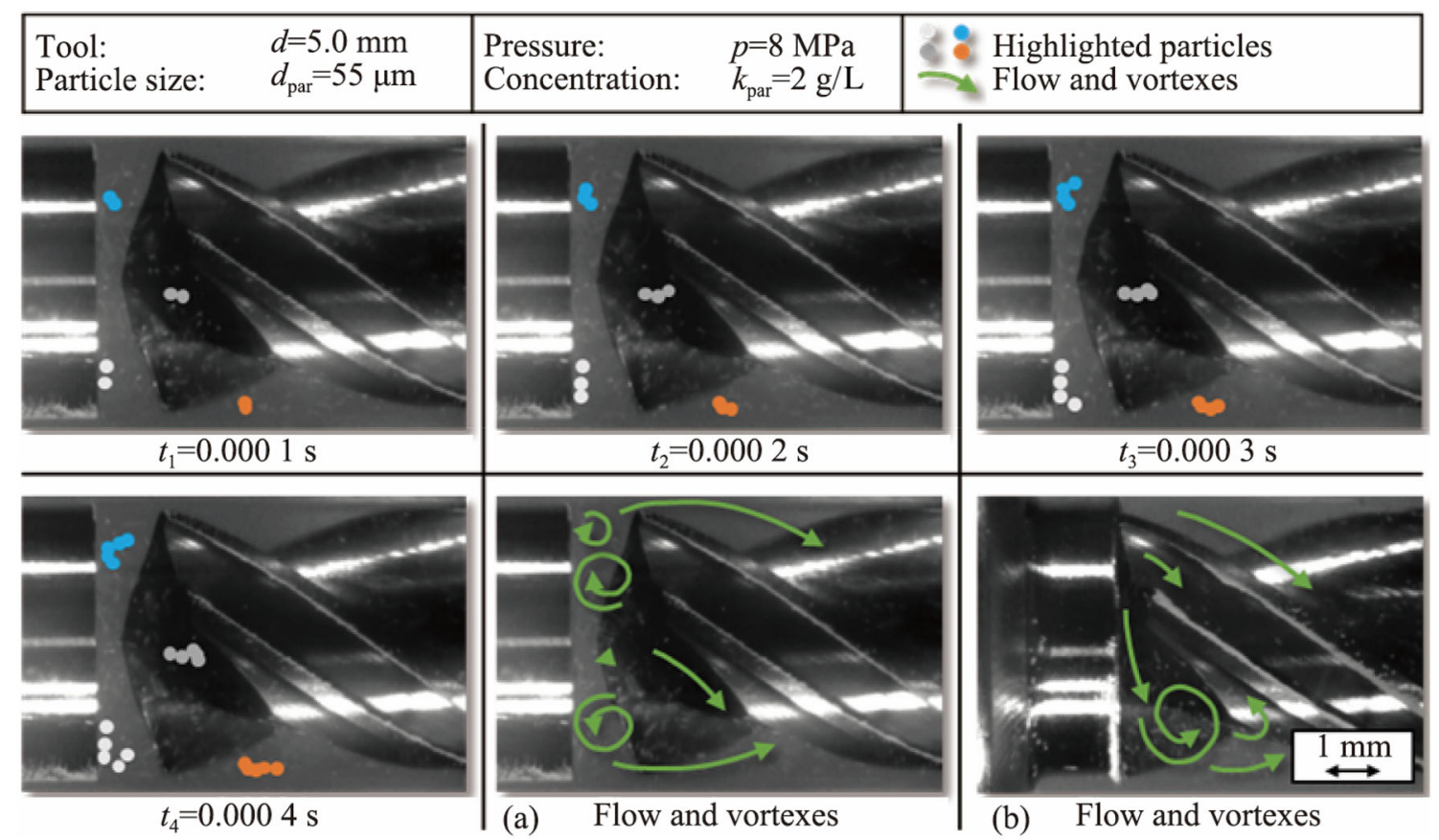

Fig. 5 Detection and tracking of micro-particles in the flow by high-speed recordings from $t_{1}$ to $t_{4}$. The trajectories of different micro-particles are marked in different colors. The flow behavior and the vortexes are shown a without contact of tool and workpiece and $\mathbf{b}$ for the position with full engagement of the drill

such as the geometric definition of the boundary conditions, the resistance coefficients, the driving forces and the interactions, as well as the input and output parameters of the flow have to be defined in advance of the CFD modeling. Hence, a realistic representation of the flow is obtained. In addition to that, the correct selection of the turbulence model plays an important role for the CFD simulation. Conventional CFD simulations often use the properties of water to describe incompressible fluids. A special feature of the CFD simulation in this paper is the adaptation of the fluid parameters to the viscous properties of the cutting fluid. The RANS $k$ - $\omega$-SST turbulence model 


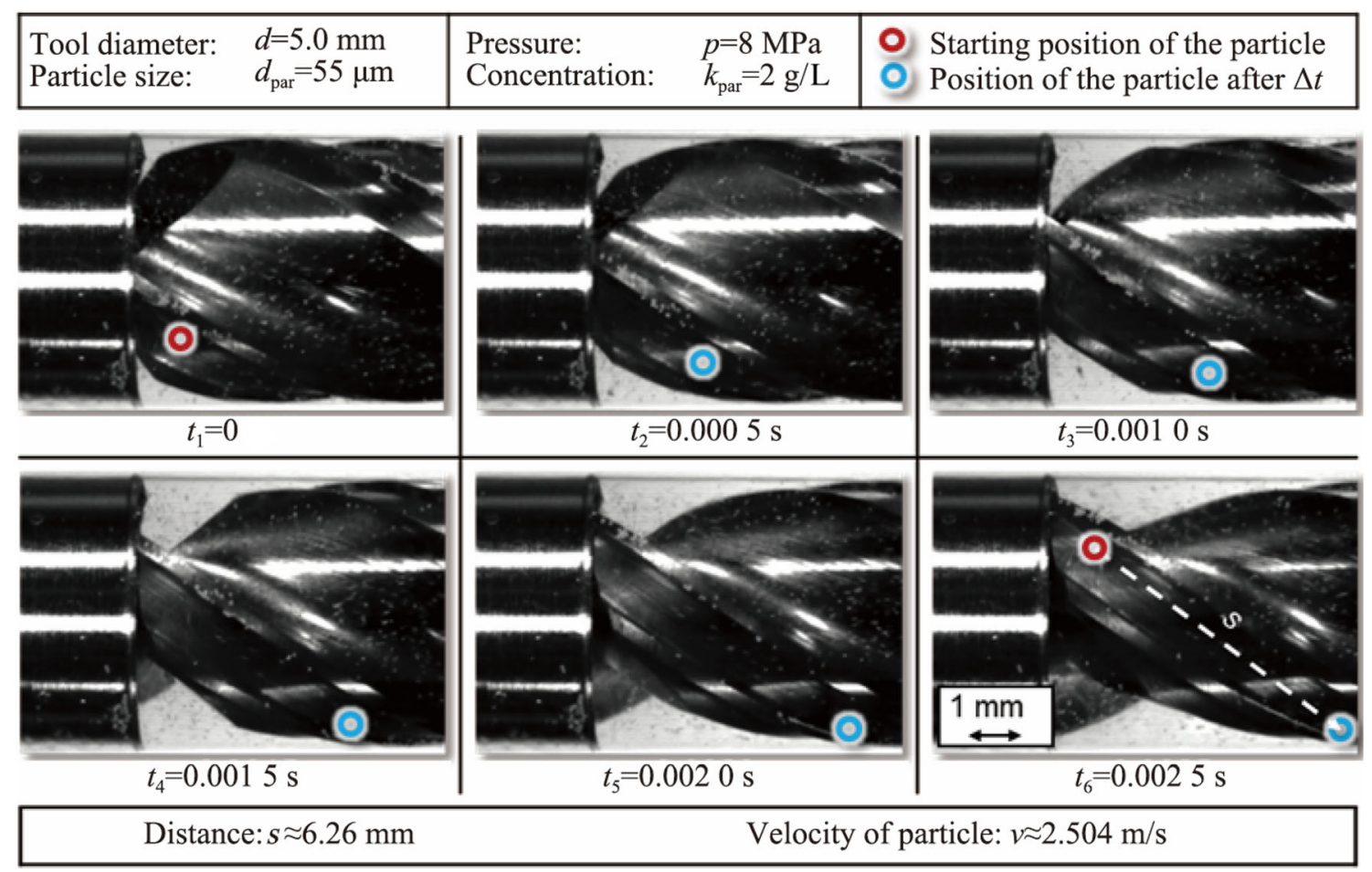

Fig. 6 Flow velocity analysis by tracking an individual micro-particle as well as calculating the distance of starting position $t_{1}$ and end position $t_{6}$

was used to carry out the simulation since it proved to be the most suitable in comparison with the standard $k-\omega$ model and the $k-\varepsilon$ model [31]. Since turbulences, especially on a small scale, dissolve very differently in space and time, extremely fine grids and simulation time steps are required to achieve reliable results with the CFD analysis. This is of great interest especially in the field of metal cutting because the use of CFD in particular holds a high potential for tool optimization. The precise knowledge of the flow behavior as well as the consideration of the viscous fluid properties enable the implementation of resultoriented improvements for the design of drilling tools. The boundary conditions of the CFD simulation are listed in Table 2 .

\subsection{Simulation results and validation with experimental values}

The CFD simulation was carried out in ANSYS CFX/ FLUENT, which is based on the finite volume method (FVM). The results of the CFD simulation were validated with experimental data to evaluate the achieved model quality.

\subsubsection{Evaluation of the numerically determined flow behavior}

To validate the values of the numerical determined flow velocities an averaged flow velocity, corresponding to the measurements of the experiment, was calculated. In the two sectional planes $A-A$ (start position, $t_{1}=0$ ) and $B$ $B$ (end position, $t_{6}=0.0025 \mathrm{~s}$ ) with a distance of $s=6.26 \mathrm{~mm}$ six values for the flow velocity between the guide chamfers of the helical drill were determined to calculate an arithmetic average (see Fig. 7).

The resulting arithmetic average of the flow velocity in the sectional plane $A-A\left(v_{\mathrm{A} 1}\right.$ to $\left.v_{\mathrm{A} 6}\right)$ is $v_{\mathrm{A}}=2.605 \mathrm{~m} / \mathrm{s}$, and in the sectional plane $B-B\left(v_{\mathrm{B} 1}\right.$ to $\left.v_{\mathrm{B} 6}\right)$ the arithmetic average is $v_{\mathrm{B}}=2.480 \mathrm{~m} / \mathrm{s}$. In the sectional plane $A$ $A$ higher fluid velocities occur than in the further course of the flow between the guide chamfers, which can be attributed to the higher fluid momentum in the vicinity of the bore hole bottom. The total arithmetic average of the simulated flow velocity was $v_{\text {sim }}=2.544 \mathrm{~m} / \mathrm{s}$ (see Table 3 ). Compared to the experimental average flow velocity of $v_{\exp }$ $=2.504 \mathrm{~m} / \mathrm{s}$ (see Fig. 6), there is only a marginal difference of $1.57 \%$, which indicates a very good model quality.

Based on the validation of the simulated values between the guide chamfers, the results in Fig. 7 demonstrate that even in the flutes of the helical drill the flow velocities are very low with approximately $v_{\text {oil }}=27 \mathrm{~m} / \mathrm{s}$. It can be seen 
Table 2 Boundary conditions of the CFD simulation (helical deep hole drill with internal coolant channels)

\begin{tabular}{|c|c|c|}
\hline Simulation condition & Variable or type & Value or model \\
\hline Calculation type: transient & Total simulation time & $t=4 \mathrm{~s}$ \\
\hline Simulation time steps & Time step & $t=0.0135 \mathrm{~s}$ \\
\hline \multirow[t]{8}{*}{ Fluid } & Type & Swisscut Ortho NF-X \\
\hline & Kinematic viscosity & $v_{k}=10.9 \mathrm{~mm}^{2} / \mathrm{s}\left(40^{\circ} \mathrm{C}\right)$ \\
\hline & Specific heat capacity & $c=2053.67 \mathrm{~J} /(\mathrm{kg} \cdot \mathrm{K})$ \\
\hline & Reference temperature & $T=25^{\circ} \mathrm{C}$ \\
\hline & Dynamic viscosity & $\mu=0.01714 \mathrm{~kg} /(\mathrm{m} \cdot \mathrm{s})$ \\
\hline & Thermal conductivity & $\lambda=0.124 \mathrm{~W} /(\mathrm{m} \cdot \mathrm{K})$ \\
\hline & Density & $\rho=857 \mathrm{~kg} / \mathrm{m}^{3}$ \\
\hline & Molar mass & $M=370 \mathrm{~g} / \mathrm{mol}$ \\
\hline \multirow[t]{2}{*}{ Inlet } & Pressure & $p=8 \mathrm{MPa}$ \\
\hline & Mass flow & $\dot{m}=0.7 \mathrm{~kg} / \mathrm{s}$ \\
\hline Outlet: ambient pressure & Pressure & $p=0.1 \mathrm{MPa}$ \\
\hline Interface 1: workpiece/fluid & Wall roughness & $R_{z}=16 \mu \mathrm{m}$ \\
\hline Interface 2: tool/fluid & Wall roughness & Smooth \\
\hline Interface 1,2 & Rotational speed & $n=4556 \mathrm{r} / \mathrm{min}$ \\
\hline Turbulence model & Type & RANS $k$ - $\omega$-SST \\
\hline Fluid model & Number of elements & ca. 71 million \\
\hline Inflation-layer: first layer & Length & $1.0 \times 10^{-3} \mathrm{~mm}$ \\
\hline Inflation-layer: growth rate & Factor & 1.2 \\
\hline
\end{tabular}

that in local areas, especially between the guide chamfers, the velocity drops to almost $v_{\text {oil }}=0$. In the flow itself, most of the fluid movement can be attributed to the tool rotation, resulting in an unfavorable distribution of the fluid, especially in the cutting zone and directly at the cutting edge.

\subsubsection{Turbulence intensity}

Turbulences are a very complex phenomenon. Large turbulent vortexes extract kinetic energy from the main flow by decomposing from large-scale structures to smaller structures. This phenomenological process is called the Kolmogorov's energy cascade. The anisotropic movement causes shear forces between the energy-saving vortexes, so that the turbulence elements become smaller and smaller and dissipate the kinetic energy into heat in the Kolmogorov range, thus withdrawing kinetic energy from the flow. As soon as the large vortexes are generated continuously and interact non-linearly with each other, large flow fluctuations occur, i.e., the velocities can fluctuate very strongly. The turbulence intensity is a measure of the strength of the turbulences and has great importance in fluid mechanics. In order to assess the degree of fluctuation of the flow, the so-called degree of turbulence turbulence intensity (TI) is defined by the ratio of the turbulent velocity fluctuations $V_{\mathrm{RMS}}$ to the mean velocity $v$ [42]
$\mathrm{TI}=\frac{V_{\mathrm{RMS}}}{v}$.

The standard deviation of the turbulent velocity fluctuation $V_{\text {RMS }}$ represents an alternative measure for the temporal fluctuations at a particular location over a specified period of time. The average of the velocity $v$ is calculated at the same location over the same time period. For velocity components with a time-averaged velocity, $V_{\mathrm{RMS}}$ can be calculated as

$V_{\mathrm{RMS}}=\sqrt{\frac{1}{3}\left(\left(V_{\mathrm{RMSu}}\right)^{2}+\left(V_{\mathrm{RMS}}\right)^{2}+\left(V_{\mathrm{RMS}_{\mathrm{w}}}\right)^{2}\right)}$.

Thereby, the fluctuating velocity components are in a mutually perpendicular coordinate system $(u, v, w)$. The kinetic turbulence energy is physically defined by the rootmean-square (RMS) and will be calculated with the turbulence model [43]. The total mass-related energy contained in the turbulence movements is referred to as the turbulent kinetic energy $k$, which is defined to be half of the variances of the velocity components and can be determined as

$k=\frac{1}{2}\left(\left(V_{\mathrm{RMSu}}\right)^{2}+\left(V_{\mathrm{RMS}_{\mathrm{v}}}\right)^{2}+\left(V_{\mathrm{RMS} \mathrm{w}}\right)^{2}\right)$.

To determine the isotropic turbulence the specified values of $k$ are approximated. In this case the final equation 


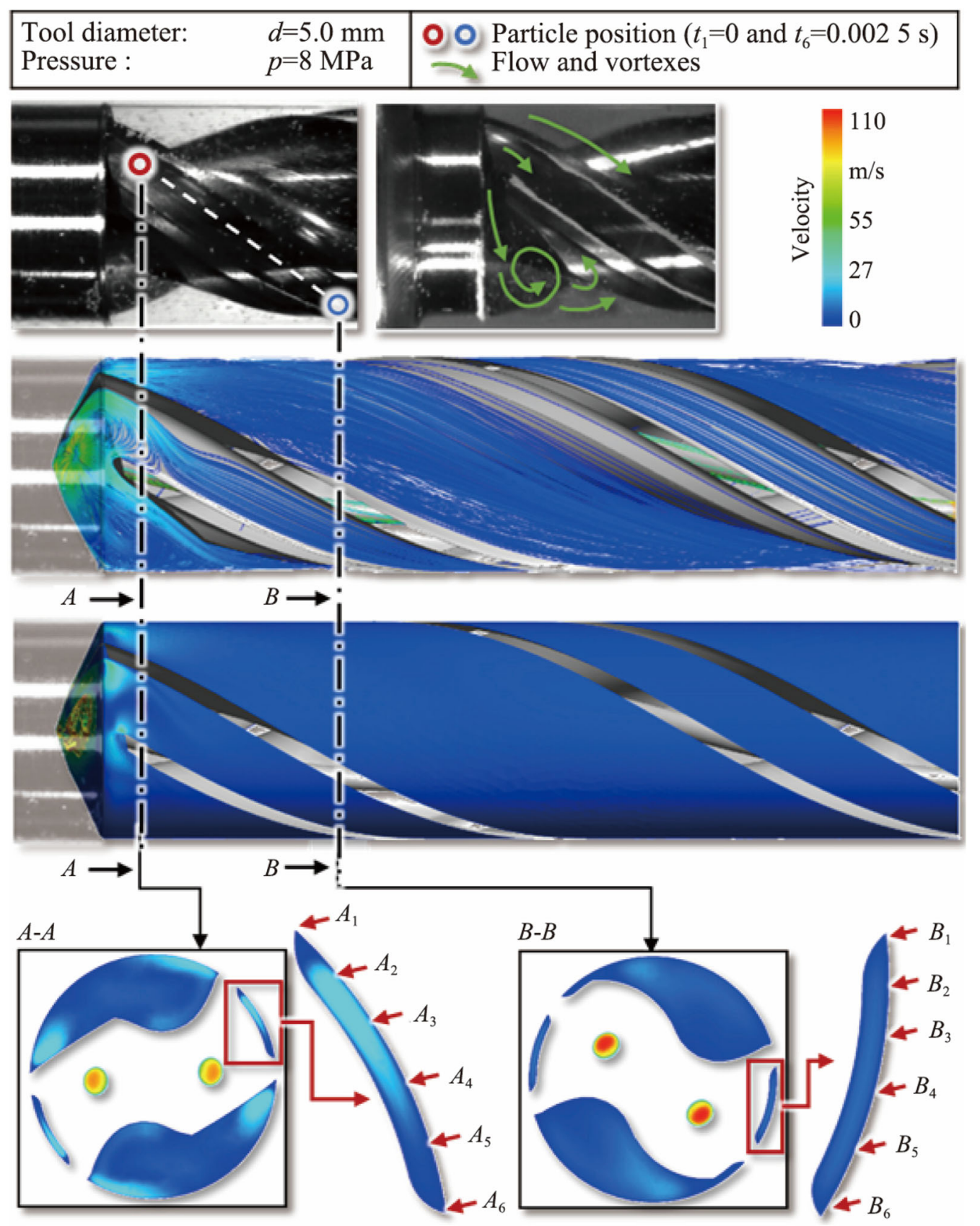

Fig. 7 Numerical evaluation of the flow velocities corresponding to the experimental observation of the micro-particle movement

for the isotropic turbulence is derived from the combination of Eqs. (16), (17)

$V_{\mathrm{RMS}}=\sqrt{\frac{2}{3}} k$

The point at which turbulences first occur is generally influenced by three critical variables: flow velocity, pipe diameter and fluid viscosity. This means that the faster the fluid flows and the further away the calming and guiding pipe wall is from the center of the flow, the easier vortexes are formed. In addition, the more viscous the liquid, the more likely vortexes and thus turbulences are slowed down. The cutting fluid flows through the two cooling channels of the tool at a high velocity of approximately $v=110 \mathrm{~m} / \mathrm{s}$ in the middle of the cooling channel and at a much lower velocity close to the walls. On the one hand, the closer the flow gets to the contact zone between cutting edge and bore hole ground after the cutting fluid exits the cooling channels of the tool in order to lubricate the contact zone, the lower the flow velocity. Thus, the vortex formation decreases as well. On the other hand, the viscous flow properties and vortexes formation in this area are 
Table 3 List of the simulated values of the arithmetic average flow velocity

\begin{tabular}{llll}
\hline $\begin{array}{l}\text { Sectional view } \\
A-A\end{array}$ & $\begin{array}{l}\text { Average } \\
\text { velocity }\end{array}$ & $\begin{array}{l}\text { Sectional view } \\
B-B\end{array}$ & $\begin{array}{l}\text { Average } \\
\text { velocity }\end{array}$ \\
\hline$v_{\mathrm{A} 1}=0.92 \mathrm{~m} / \mathrm{s}$ & $v_{\mathrm{A}}=2.605 \mathrm{~m} / \mathrm{s}$ & $\begin{array}{l}v_{\mathrm{B} 1}=2.44 \mathrm{~m} / \mathrm{s} \\
v_{\mathrm{B} 2}=5.76 \mathrm{~m} / \mathrm{s}\end{array}$ & $v_{\mathrm{B} 2}=2.49 \mathrm{~m} / \mathrm{s}$ \\
$v_{\mathrm{A} 3}=3.64 \mathrm{~m} / \mathrm{s}$ & $v_{\mathrm{B} 3}=2.57 \mathrm{~m} / \mathrm{s}$ & \\
$v_{\mathrm{A} 4}=2.81 \mathrm{~m} / \mathrm{s}$ & $v_{\mathrm{B} 4}=2.62 \mathrm{~m} / \mathrm{s}$ & \\
$v_{\mathrm{A} 5}=1.43 \mathrm{~m} / \mathrm{s}$ & $v_{\mathrm{B} 5}=2.36 \mathrm{~m} / \mathrm{s}$ \\
$v_{\mathrm{A} 6}=1.07 \mathrm{~m} / \mathrm{s}$ & $v_{\mathrm{B} 6}=2.42 \mathrm{~m} / \mathrm{s}$ & \\
\hline
\end{tabular}

supported by the high temperatures generated in the contact zone during drilling. As mentioned above, the movement of the flow results almost exclusively from the rotation of the tool. Due to the low kinetic energy in the cutting fluid, the vortexes tend to decompose quickly. The already low flow velocity of the cutting fluid at the cooling channel outlet is additionally slowed down in the narrow contact area at the cutting edge and by the chaotic decomposing vortices and drops to almost $v=0$.

The turbulent fluctuations of the flow are described quantitatively by the turbulence energy and cause a change of the physical properties within the flowing fluid. The fluctuations of the flow velocity and the flow pressure are subjected to dissipation in this area, which also depends on the viscosity of the fluid. Since the processes are slow compared to the main flow and the fluctuation velocities are dominant for turbulent flows, their effect remains local but has an enormous influence on the flow process near the cutting edge of the tool. Due to the high turbulence intensity and the low dissipation, the flow velocity and the flow pressure in the immediate vicinity of the cutting edge decrease to such an extent that the fluid cannot generate sufficient cooling or lubrication (see Fig. 8).

\section{Summary and conclusion}

This study presents the experimental and computational analysis of the cooling and lubrication distribution under consideration of the viscosity of the cutting fluid during deep hole drilling. The flow behavior and the interactions of the cutting fluid with a kinematic viscosity of $v_{\mathrm{k}}=10.9$ $\mathrm{mm}^{2} / \mathrm{s}\left(40^{\circ} \mathrm{C}\right)$ were investigated. The workpiece material was AISI 316L and the deep hole drill had a diameter of $d=5 \mathrm{~mm}$. With a developed high-pressure mixing vessel, tracer micro-particles could be fed into the cutting fluid circuit and the flow behavior could thus be visualized using high-speed microscopy. A particle diameter of $d_{\text {par }}=55$ $\mu \mathrm{m}$ and a concentration of $k_{\mathrm{par}}=2 \mathrm{~g} / \mathrm{L}$ were suitable for individual particle detection. Since in the context of preliminary investigations small variations of the cutting fluid pressure did not significantly affect the process, a pressure of $p=8 \mathrm{MPa}$, corresponding to common industrial applications, was chosen. Due to the limited frame rate of the high-speed camera and higher flow velocities in the chip flutes, the flow velocity was experimentally determined between the guide chamfers of the tool. Within a period of $\Delta t=0.0025 \mathrm{~s}$ the particle and thus the fluid was transported over a distance of approximately $s \approx 6.26 \mathrm{~mm}$ resulting in an average flow velocity of $v_{\text {oil }}=2.504 \mathrm{~m} / \mathrm{s}$. The obtained results were used to validate a CFD simulation, enabling the analysis of the flow in areas that cannot be assessed experimentally due to the inaccessibility of the cutting zone. Contrary to conventional numerical calculations the properties of the cutting fluid, i.e., the temperature-dependent kinematic viscosity, have been considered in this study. For the numerical analysis of the flow behavior boundary conditions were defined and a fluid model was accurately meshed. The $k$ - $\omega$-SST turbulence model, which combined the advantages of the $k-\varepsilon$ and $k-\omega$ models, was used. For the validation of the numerical resulted an averaged flow velocity, corresponding to the measurements of the experiment, was calculated. The results showed a marginal difference of $1.57 \%$ between simulation and experiment proving the excellent model quality. The cutting fluid exits the tool's cooling channels with a velocity of approximately $v=110 \mathrm{~m} / \mathrm{s}$. The fluid is then distributed in the space between the tool and the workpiece. The results of the simulation show that the fluid velocity drops to almost $v=0$ in local areas especially directly at the cutting edge as well as between the guide chamfers. The turbulence intensity with an average value of $\mathrm{TI}=0.2 \%$ is very low. As a consequence, the flow pressure close to the cutting edge decrease by approximately $35 \%$. This results in insufficient lubrication and cooling of the guide chamfers and cutting edges, which promotes rapid tool wear.

In order to achieve an efficient flow distribution, the tool geometry and the process need to be optimized to realize higher flow velocities and higher flow pressures. Due to the small tool diameter, an increase in the cooling channel diameter is not possible, as it weakens the tool and process reliability can no longer be guaranteed. During the experiments a simple increase in pressure did not show any improvement of the flow behavior in the critical areas at the cutting edge. Another possibility to achieve a better flow behavior and higher flow velocities could be to change the tool geometry by adapting the flank face of the tool as well as to optimize the surface of the cooling channels. With additive manufacturing processes, internal helixes or rib-like notches can be realized to generate turbulences and accelerate the flow. The CFD simulation considering the 


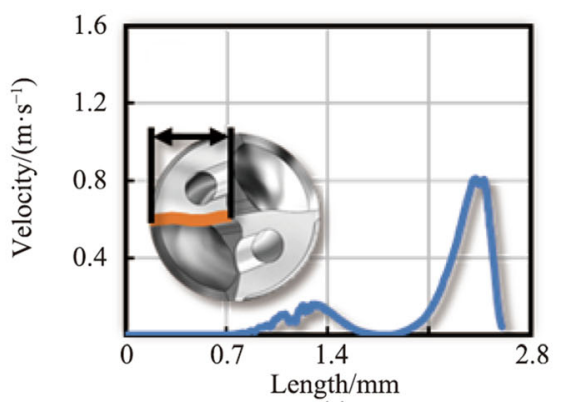

(a)

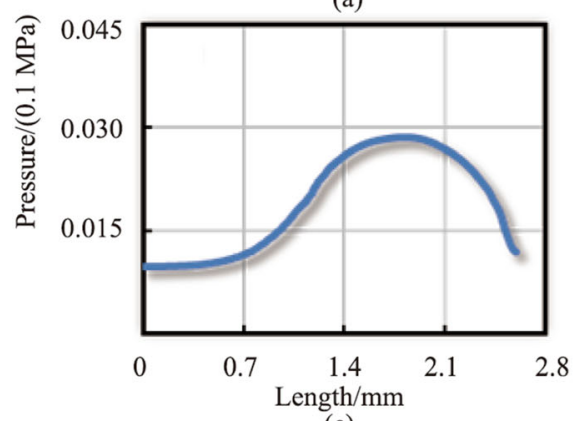

(c)

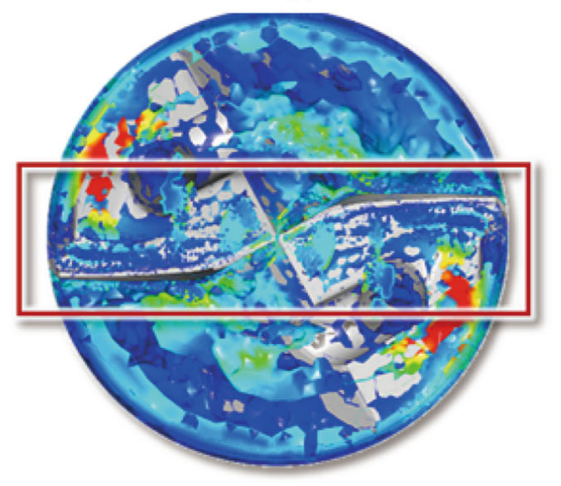

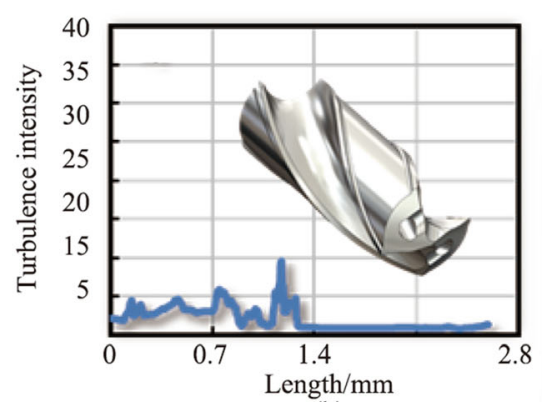

(b)

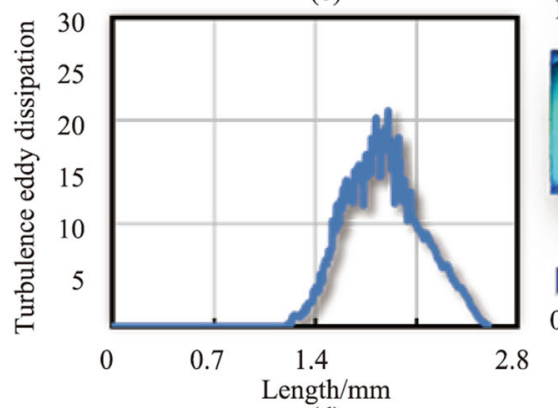

(d)
$Q$ criterion: turbulence model $k$ - $\omega$-SST

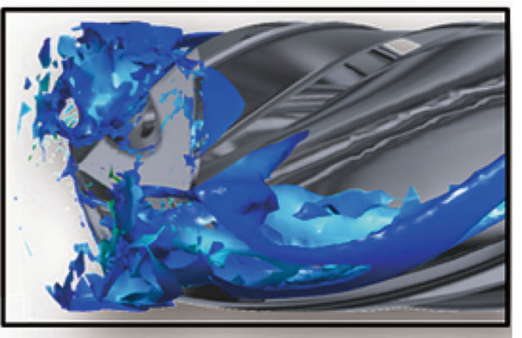

Fully developed fiow

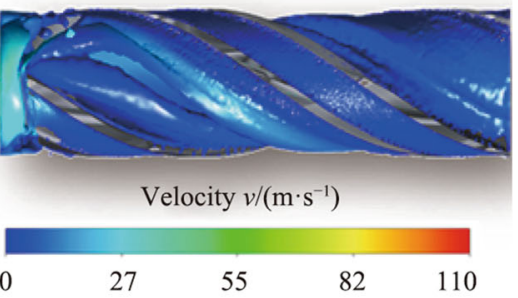

8

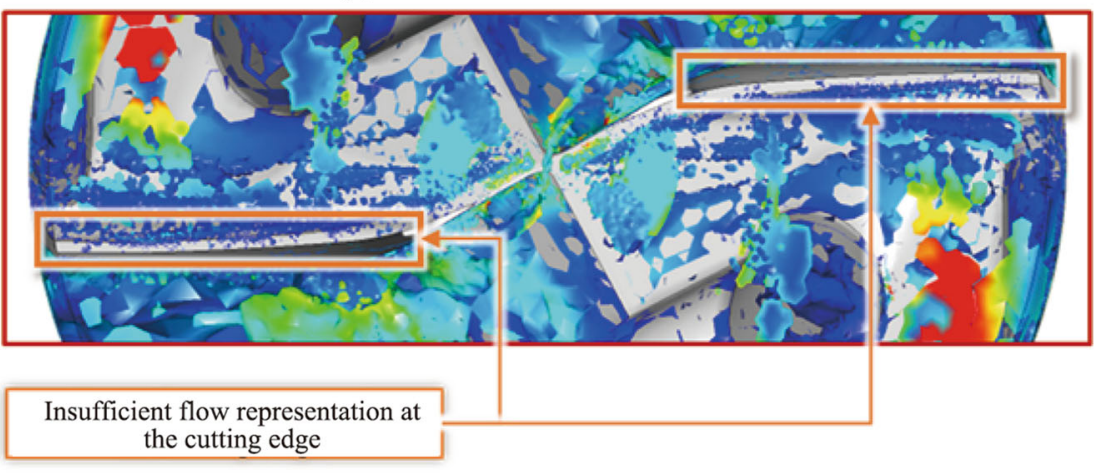

Fig. 8 Results of the CFD simulation a flow velocity, $\mathbf{b}$ turbulence intensity, $\mathbf{c}$ flow pressure, $\mathbf{d}$ turbulence eddy dissipation

viscous properties of the cutting fluid can be used to rapidly evaluate the coolant efficiency of these changes in the tool design and help to optimize the tool geometry. For future investigations, suitable mathematical models must be developed so that the strongly temperature- and pressuredependent dynamic viscosity can be taken into account in the simulation. This makes it possible to evaluate different viscous properties and adjust the cutting fluid properties exactly to the tool and the drilling process. However, it must always be considered that the temperatures during the machining process are also depending on tool geometry, cutting parameters and workpiece material.

Acknowledgements The authors gratefully acknowledge funding from the Deutsche Forschungsgemeinschaft (DFG, German Research Foundation) for the research project (Grant No. 317373968).

Open Access This article is licensed under a Creative Commons Attribution 4.0 International License, which permits use, sharing, adaptation, distribution and reproduction in any medium or format, as long as you give appropriate credit to the original author(s) and the source, provide a link to the Creative Commons licence, and indicate if changes were made. The images or other third party material in this article are included in the article's Creative Commons licence, unless indicated otherwise in a credit line to the material. If material is not included in the article's Creative Commons licence and your intended use is not permitted by statutory regulation or exceeds the permitted use, you will need to obtain permission directly from the copyright holder. To view a copy of this licence, visit http://creativecommons. org/licenses/by/4.0/.

\section{References}

1. Aamir M, Giasin K, Tolouei-Rad M et al (2020) A review: drilling performance and hole quality of aluminium alloys for aerospace applications. J Mater Res Tech 9:12484-12500

2. Abele E, Fujara M (2010) Simulation-based twist drill design and geometry optimization. CIRP Ann-Manuf 59:145-150

3. Kyratsis P, Bilalis N, Antoniadis A (2011) CAD-based simulations and design of experiments for determining thrust force in drilling operations. Comput Aided Design 43:1879-1890

4. Luttervelt CA, Childs THC, Jawahir IS et al (1998) Present situation and future trends in modelling of machining operations 
progress report of the cirp working group 'modelling of machining operations'. CIRP Ann-Manuf 47:587-626

5. Mackerle J (1999) Finite-element analysis and simulation of machining: a bibliography (1976-1996). J Mater Process Tech $86: 17-44$

6. Mackerle J (2003) Finite element analysis and simulation of machining: an addendum a bibliography (1996-2002). Int J Mach Tools Manuf 43:103-114

7. Arrazola PJ, Özel T, Umbrello D et al (2013) Recent advances in modelling of metal machining processes. CIRP Ann-Manuf 62:695-718

8. Aurich JC, Bil H (2006) 3D finite element modelling of segmented chip formation. CIRP Ann-Manuf 55:47-50

9. Heinemann R, Hinduja S, Barrow G et al (2006) The performance of small diameter twist drills in deep-hole drilling. J Manuf Sci Eng 128:884-892

10. Abukhshim NA, Mativenga PT, Sheikh MA (2006) Heat generation and temperature prediction in metal cutting: a review and implications for high speed machining. Int J Mach Tools Manuf 46:782-800

11. Çakīr O, Yardimeden A, Ozben T et al (2007) Selection of cutting fluids in machining processes. J Achieve Mater Manuf Eng 25:99-102

12. Fallenstein F, Aurich JC (2014) CFD based investigation on internal cooling of twist drills. Proc CIRP 14:293-298

13. Bücker M, Oezkaya E, Hensler U et al (2020) A new flank face design leading to an improved process performance when drilling high-temperature nickel-base alloys. MIC Procedia. http://doi. org $/ 10.2139 /$ ssrn. 3722782

14. Uçak N, Çiçek A (2018) The effects of cutting conditions on cutting temperature and hole quality in drilling of Inconel 718 using solid carbide drills. J Manuf Process 31:662-673

15. Verein DI (2014) VDI-Richtlinie 3210: Tiefbohrverfahren. Beuth Verlag, Berlin

16. Biermann D, Bleicher F, Heisel U et al (2018) Deep hole drilling. CIRP Ann-Manuf 67:673-694

17. Uhlmann E, Richarz S (2016) Twisted deep hole drilling tools for hard machining. J Manuf Processes 24:225-230

18. Khan SA, Nazir A, Mughal MP et al (2017) Deep hole drilling of AISI 1045 via high-speed steel twist drills: evaluation of tool wear and hole quality. Int J Adv Manuf Technol 93:1115-1125

19. Volz M, Abele E, Weigold M (2020) Lateral vibrations in deep hole drilling due to land width variation. J Manuf Mater Process 28:1-14

20. Kirschner M, Michel S, Berger S et al (2017) Adaption of the tool design in micro deep hole drilling of difficult-to-cut materials by high-speed chip formation analyses. In: Schmitt R, Schuh G (eds) 7, Apprimus Verlag, WGP-Jahreskongress Aachen, pp 29-36

21. Fettig A (2021) Deep hole drilling demands precise coolant control. Manuf Eng 6:12-14

22. Mihail LA (2013) Robust engineering of deep drilling process by surface state optimization. Proc CIRP 8:582-587

23. Heinemann RK, Hinduja S (2009) Investigating the feasibility of DLC-coated twist drills in deep-hole drilling. Int J Adv Manuf Technol 44:862-869

24. Oezkaya E, Michel S, Biermann D (2018) Experimental studies and FEM simulation of helical-shaped deep hole twist drills. Prod Eng Res Devel 12:11-23

25. Schnabel D, Oezkaya E, Biermann D et al (2017) Modeling the motion of the cooling lubricant in drilling processesusing the finite volume and the smoothed particle hydrodynamics methods. Comput Method Appl M 329:369-395

26. Biermann D, Iovkov I, Blum H et al (2012) Thermal aspects in deep hole drilling of aluminium cast alloy using twist drills and MQL. Proc CIRP 3:245-250
27. Wosniak FA, Polli ML, Camargo BPA (2016) Study on tool wear and chip shapes in deep drilling of AISI 4150 steel. J Braz Soc Mech Sci Eng 38:473-480

28. Aized T, Amjad M (2013) Quality improvement of deep-hole drilling process of AISI D2. Int $\mathbf{J}$ Adv Manuf Technol 69:2493-2503

29. Wegert R, Guski V, Möhring HC et al (2020) Temperature monitoring in the subsurface during single lip deep hole drilling. tm-Technisches Messen 87:757-767

30. Schmidt R, Strodick S, Walther F et al (2020) Analysis of the functional properties in the bore sub-surface zone during BTA deep-hole drilling. Proc CIRP 88:318-323

31. Beer N, Oezkaya E, Biermann D (2014) Drilling of Inconel 718 with geometry-modified twist drills. Proc CIRP 9:49-55

32. Raffel M, Willert CE, Scarano F et al (2018) Particle image velocimetry. Springer International Publishing, Cham

33. Westerweel J, Elsinga GE, Adrian RJ (2013) Particle image velocimetry for complex and turbulent flows. Annu Rev Fluid Mech 45:409-436

34. Blazek J (2015) Computational fluid dynamics principles and applications. Heinemann, Butterworth

35. Chorin AJ, Marsden JE (2000) A mathematical introduction to fluid mechanics, 3rd edn. Springer, New York

36. Fletcher CAJ (2006) Computational techniques for fluid dynamics 2, Springer, Berlin

37. Hosain L (2018) Fluid flow and heat transfer simulations for complex industrial applications. Dissertation, Mälardalen University Press, Sweden

38. Alfonsi G (2009) Reynolds-averaged navier-stokes equations for turbulence modeling. Appl Mech Rev 62:040802-040822

39. Durbin PA (2018) Turbulence closure models for computational fluid dynamics. In: Stein E, de Borst R, Hughes TJR (eds) Encyclopedia of computational mechanics, vol 63, 2nd edn, Chichester, UK, pp 1-22

40. Menter FR (1994) Two-equation eddy-viscosity turbulence models for engineering applications. AIAA J 32:1598-1605

41. Wilcox DC (1988) Reassessment of the scale-determining equation for advanced turbulence models. AIAA J 26:1299-1310

42. Russo F, Basse NT (2016) Scaling of turbulence intensity for low-speed flow in smooth pipes. Flow Meas Instrum 52:101-114

43. Markatos NC (1986) The mathematical modelling of turbulent flows. Appl Math Model 10:190-220

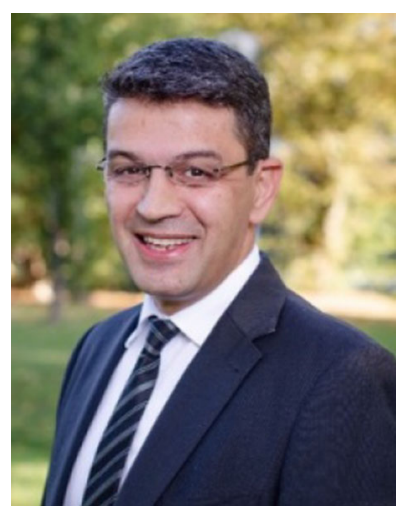

Dr.-Ing. Ekrem Oezkaya has been a Research Fellow at the Institute of Machining Technology at TU Dortmund University since 2011. His research interests are in various interdisciplinary fields, such as machining and manufacturing processes. His expertise lies in the developments by innovative simulative and mathematical solutions, models for optimization procedures and novel CAD solutions via application programming interfaces, computational fluid dynamics (CFD) simulation, finite element method (FEM) simulation, fluid structure interface (FSI) simulation, and the investigation of modern difficult to machine materials. 


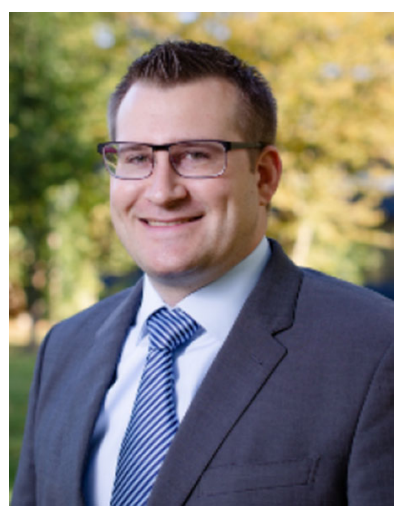

Sebastian Michel M. Sc. has been a research assistant at the Institute of Machining Technology TU Dortmund University since 2016. His research areas concentrate on the development and optimization of single-lip and twist drill deep hole drilling tools with focus on small tool diameters and difficult-to-cut materials and the machining of fiber composite materials by drilling and milling processes.

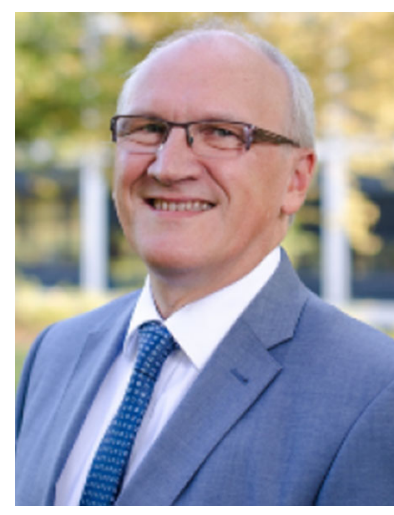

from industry and academia.
Prof. Dr.-Ing. Prof. H. C. Dirk

Biermann is the director of the Institute for Machining Technology at the Technical University of Dortmund. His research areas cover all relevant machining processes and the information technology environment in machining. In addition, various modelling concepts, as well as optimization in production technology are the focus of his scientific work, which are elaborated in direct cooperation with partners 